\title{
Reanálisis de la diversidad alfa: alternativas para interpretar y comparar información sobre comunidades ecológicas
}

\section{Reanalyzing alpha diversity: alternatives to understand and compare information about ecological communities}

\author{
Claudia E. Moreno ${ }^{1 凶}$, Felipe Barragán ${ }^{1}$, Eduardo Pineda² y Numa P. Pavón ${ }^{1}$ \\ ${ }^{1}$ Centro de Investigaciones Biológicas, Universidad Autónoma del Estado de Hidalgo. Apartado. postal 69-1, 42001 Pachuca, Hidalgo, México. \\ ${ }^{2}$ Red de Biología y Conservación de Vertebrados, Instituto de Ecología, A.C. Carretera antigua a Coatepec 351, El Haya 91070 Xalapa, Veracruz, \\ México. \\ \cmoreno@uaeh.edu.mx
}

\begin{abstract}
Resumen. El índice de entropía de Shannon y otras medidas de complejidad se utilizan frecuentemente para evaluar la diversidad de especies en comunidades ecológicas, aun cuando su comprensión es difícil y sus valores no son comparables. En este trabajo se muestra que los números efectivos de especies (medidas de diversidad verdadera) permiten obtener una interpretación intuitiva y fácilmente comparable de la diversidad de especies. Se ejemplifica su uso reanalizando los datos de 4 trabajos publicados en la Revista Mexicana de Biodiversidad (realizados en distintos ecosistemas y regiones de México, con distinta resolución taxonómica y enfocados en distintos grupos biológicos). Se utilizan modelos de estimación en los que se considera que las muestras son representaciones incompletas de las comunidades. Se explica también la manera en que las medidas de diversidad de distinto orden incorporan a las especies según su abundancia en la comunidad. Los resultados obtenidos pueden resultar de especial interés cuando los valores de diversidad se utilizan para proponer medidas para el manejo de recursos y la conservación biológica.
\end{abstract}

Palabras clave: diversidad verdadera, especies equivalentes, especies igualmente comunes, índice de Shannon, riqueza, equidad, dominancia, abundancia.

\begin{abstract}
The Shannon index of entropy and related measures of compositional complexity are often used to assess species diversity in ecological communities, even when they are difficult to understand and their values are not comparable. This paper shows that the effective numbers of species (true diversity measures) allows for a more intuitive interpretation, and offers easily comparable values of species diversity. Their use is exemplified by reanalyzing the data from 4 studies published in the Revista Mexicana de Biodiversidad (done in different ecosystems and regions of Mexico, with different levels of taxonomic resolution and different biological groups). Diversity estimation models are also used; these assume that samples are incomplete representations of communities. This paper also offers an explanation of how diversity measures of different orders incorporate species depending on their abundance in the community. This may be especially useful when the diversity values are used to support proposals for resource management and biological conservation.
\end{abstract}

Key words: true diversity, species equivalents, equally common species, Shannon index, richness, evenness, dominance, abundance.

\section{Introducción}

La diversidad de especies es un tema central tanto en ecología de comunidades como en biología de la conservación. Su estudio ha adquirido mayor relevancia en los últimos años debido a su posible relación con el funcionamiento de los ecosistemas (a través de procesos tales como la productividad y la estabilidad) y por su modificación como resultado de actividades humanas (Maclaurin y Sterelny, 2008). A pesar de su amplio uso, el concepto

Recibido: 17 agosto 2010; aceptado: 11 mayo 2011 de diversidad de especies no siempre es claro, y su definición y cuantificación han generado mucha polémica durante más de medio siglo. Desde la década de los 70 del siglo pasado, se habían detectado problemas semánticos, conceptuales y técnicos, ante los cuales se concluyó que la diversidad de especies se había convertido en un concepto sin sentido (Hurlbert, 1971), y que las medidas de diversidad disponibles eran simplemente respuestas para las cuales aún no se habían generado las preguntas (Poole, 1974). Esta inquietud se generó al tratar como sinónimos de la diversidad el fenómeno biológico y el valor obtenido mediante el cálculo de algún índice de complejidad (Pie- 
lou, 1980). Actualmente no está en discusión la existencia de la diversidad de especies como una propiedad real de las comunidades ecológicas, lo cual vale la pena evaluar (Podani, 2006). Tal vez no sea una propiedad inequívoca e inherente (Molinari, 1989; Ricotta, 2005), pero los distintos significados del término diversidad pueden ser útiles bajo diferentes perspectivas.

Desde el enfoque de la ecología de comunidades, la diversidad de especies es esencialmente un concepto relacionado con la estructura de las comunidades: el recíproco de un promedio de las abundancias relativas (Hill, 1973). Por lo tanto, teóricamente, si en una comunidad todas las especies tienen exactamente la misma abundancia (i.e. son igualmente comunes, o equivalentes en número), entonces la diversidad debe ser proporcional al número de especies (riqueza específica). Lo que es necesario enfatizar es que las herramientas de medición aplicables al concepto de diversidad no deben confundirse con las teorías que las soportan (Hill, 1973).

Queremos o necesitamos medir la diversidad porque, como en cualquier ciencia, las medidas permiten describir los componentes del sistema bajo estudio, hacer comparaciones entre sistemas y porque representan la materia prima para generar teorías (Maclaurin y Sterelny, 2008). Así, en ecología de comunidades necesitamos medidas apropiadas de la diversidad para generar y poner a prueba teorías sobre la coexistencia de las especies, los procesos dinámicos de los ecosistemas, los determinantes históricos y el impacto de las actividades humanas, entre otros temas. De igual forma, es posible comparar la magnitud de la diversidad entre 2 o más comunidades (por ejemplo, entre diferentes tipos de hábitat, en distintos momentos de tiempo, o a través de gradientes ambientales naturales o antrópicos). Además, los cambios en la magnitud de la diversidad pueden utilizarse para justificar acciones de protección de los ecosistemas. Entonces, si contamos con una definición clara del concepto de diversidad de especies, y tenemos razones apremiantes y justificadas para evaluarlo, necesitamos medidas adecuadas de diversidad.

Sin embargo, por décadas se ha generalizado el uso de índices sin un adecuado entendimiento de sus significados; por ejemplo, aquellos desarrollados en la teoría de la comunicación (como el índice de Shannon, Ulanowicz, 2001), que mide la entropía y no necesariamente la diversidad de una comunidad (Jost, 2006). Definimos entropía como el grado de incertidumbre en la identidad de la especie a la que pertenece un individuo seleccionado al azar de la comunidad; una comunidad donde todas las especies tienen la misma abundancia tendrá alta entropía, lo que se ha traducido como una alta diversidad.

Recientemente, Jost (2006) acuñó el termino diversidad verdadera (true diversity) para referirse de manera particular a medidas que conservan las propiedades intuitivamente esperadas del concepto de diversidad, como la propiedad de duplicación (véase más adelante), y para diferenciar estas medidas de la plétora de índices que se pueden encontrar en la literatura bajo el nombre de diversidad y que no cumplen con estas propiedades intuitivas. Aunque el término "verdadera" sea cuestionable y puedan proponerse otros adjetivos más adecuados (véase discusión en Jost, 2009; Moreno y Rodríguez, 2011), en la literatura reciente sobre el tema ya ha sido utilizado de esta manera. De manera relativamente rápida, se ha hecho evidente el impacto que empiezan a tener los números efectivos de especies, que son las unidades de medición de la diversidad verdadera. Por ejemplo, su cálculo ha sido ya incorporado en versiones recientes de algunos de los programas computacionales gratuitos más utilizados para la evaluación de la diversidad, como EstimateS 8.2.0 (Colwell, 2009), Partition 3.0 (Veech y Crist, 2009) y SPADE (Chao y Shen, 2010), así como en paquetes para el programa R, como Vegetarian. Asimismo, estas medidas han sido incorporadas en comentarios y revisiones conceptuales recientes sobre la diversidad de especies (e.g. Moreno y Rodríguez, 2010; Tuomisto, 2010a, 2010b). Incluso Ellison (2010), en el papel de editor de un foro recién publicado en la revista Ecology, concluye que hay un acuerdo general entre los autores de dicho foro: “...si el interés se centra en describir la diversidad de un ensamblaje, entonces los números efectivos de especies deben ser las medidas de diversidad elegidas, no la entropía".

El objetivo general de este trabajo es explicar las bases teóricas y ejemplificar el uso de los números efectivos (de especies o de cualquier otra unidad taxonómica) como medidas de diversidad en comunidades ecológicas (diversidad alfa). Se usaron los datos de 4 estudios de caso publicados en la Revista Mexicana de Biodiversidad para: 1), ejemplificar el uso e interpretación de los números efectivos cuando se requiere comparar adecuadamente la diversidad de distintas comunidades y 2), ejemplificar el uso de modelos de estimación de la diversidad en los que se acepta que las muestras son representaciones incompletas de las comunidades. Además de obtener valores más fáciles de comprender, las conclusiones de este trabajo se compararon con las de cada estudio original, resaltando con ello la importancia que tiene la selección de medidas apropiadas de diversidad, cuando los resultados se utilizan para comparar comunidades o para proponer estrategias para el manejo de recursos y la conservación biológica.

\section{Materiales y métodos}

Compilación de datos. En este trabajo se eligieron 4 artículos publicados en la Revista Mexicana de Biodi- 
versidad (Cuadro 1) como casos para ejemplificar el uso y significado de los números efectivos como medidas de diversidad. Los trabajos fueron seleccionados porque en todos ellos se analiza la diversidad, incluyen los datos originales de abundancia de cada especie en cada condición estudiada, están realizados con distintos grupos biológicos, en diferentes condiciones ecológicas, y con distinta resolución taxonómica (Cuadro 1). A continuación se describe brevemente el objetivo que cada uno de estos trabajos tuvo con respecto a la diversidad de especies que aquí reanalizamos: 1) comparar la diversidad de géneros de hongos de suelo aislados de 3 predios con diferente manejo agrícola en La Laguna, Coahuila (Samaniego-Gaxiola y Chew-Madinaveitia, 2007), 2) comparar la diversidad de moluscos dulceacuícolas de 5 sitios de la Reserva Ecológica El Edén, Quintana Roo, entre la época de sequía y la época de lluvias (Cózatl-Manzano y Naranjo-García, 2007), 3) comparar la diversidad de especies de lagartijas en 9 sitios de la región del Soconusco, Chiapas, a distinta altitud y con distinto tipo de vegetación: 1 pastizal inducido, 1 sitio de selva mediana, 1 de bosque mesófilo de montaña, 5 sitios de cafetal diversificado y 1 de cafetal con sombra monoespecífica de Inga micheliana (MacipRíos y Muñoz-Alonso, 2008) y 4) describir la diversidad de mamíferos en 4 localidades del istmo de Tehuantepec, Oaxaca (López et al., 2009). Para este último caso, en este trabajo se utilizaron solamente los datos de los murciélagos capturados con redes de niebla.

Análisis de datos. Los números efectivos como medidas de diversidad. Los datos obtenidos de los trabajos antes citados se analizaron con números efectivos (medidas de diversidad verdadera). Casi todos los índices relacionados con la diversidad de especies son funciones de la ecuación 1 (Jost, 2006):

$$
\sum_{i=1}^{S} p_{i}^{q}
$$

\section{Ecuación 1}

donde $p_{i}$ es la abundancia relativa de la especie $i$, es decir, la abundancia de la especie $i$ dividida entre la suma total de abundancias de las $S$ especies que integran la comunidad y el exponente $q$ es el orden de la diversidad (Hill, 1973; Jost, 2006; véase párrafo posterior). Los índices de diversidad se expresan en diferentes unidades, y por lo tanto sus valores son difíciles de interpretar. Por ejemplo, el índice de entropía de Shannon se expresa en unidades llamadas nats, cuando la base del logaritmo empleado es $e$; en bits, cuando la base es 2; o en decits, cuando la base es 10 (Tuomisto, 2010a). En este trabajo se usó una transformación que sí permite elaborar una interpretación unificada e intuitiva de la diversidad de especies, mediante el uso de números efectivos, llamada ${ }^{q} D$ (sensu Jost, 2006) o $N_{a}$ (sensu Hill, 1973) a través de la ecuación 2:

$$
{ }^{q} D=\left(\sum_{i=1}^{S} p_{i}^{q}\right)^{1 /(1-q)}
$$

Ecuación 2

donde ${ }^{q} D$ es la diversidad. Para una conversión sencilla de los índices comunes (índice de Shannon o de Simpson) en números efectivos, véase el cuadro 1 de Jost (2006). Por ejemplo, el tradicional índice de entropía de Shannon:

$$
H^{\prime}=-\sum_{i=1}^{S} p i \operatorname{lnp} i
$$

se convierte fácilmente en una medida de diversidad al expresarlo como ${ }^{I} D=\exp \left(H^{\prime}\right)$ (Jost, 2006).

El exponente $q$ determina la sensibilidad del índice a las abundancias relativas de las especies; es decir, la influencia que pueden tener las especies comunes o las especies raras en la medida de la diversidad. En sentido figurado, es un indicador que determina cuántas especies son consideradas en la muestra analizada, dependiendo de su nivel de rareza (Hill, 1973; Fig. 1). Por ejemplo, la diversidad de orden cero $(q=0)$ es completamente insensible a las abundancias de las especies; por lo tanto, el valor obtenido equivale simplemente a la riqueza de especies (Fig. 1). Los valores de $q$ menores de 1 sobrevaloran las especies raras; cuando $q=1$, todas las especies son incluidas con un peso exactamente proporcional a su abundancia en la comunidad; los valores de $q$ mayores a 1, toman más en cuenta las especies comunes (Hill, 1973; Fig. 1). Aunque $q$ puede tomar cualquier valor por ser una medida continua, no es prudente utilizar cualquier número (Hill, 1973). Lo aconsejable es reconocer cuál aspecto de la distribución de frecuencias se desea analizar, dependiendo de las características del fenómeno estudiado, y, posteriormente, seleccionar alguna de las posibilidades (Fig. 1).

La diversidad medida con números efectivos cumple, entre otros requerimientos matemáticos, con la propiedad de duplicación descrita por Hill (1973) y explicada también en los trabajos de Jost (2006, 2007, 2010). Imaginemos una comunidad A con $S$ especies cuyas abundancias relativas son $p_{i}=p_{1} \ldots p_{S}$. Si dividimos a cada una de estas $S$ especies en 2 grupos repartiendo su abundancia justo a la mitad $\left(p_{i} / 2\right)$, y luego consideramos a cada grupo como una especie distinta, tendríamos una nueva comunidad B. Intuitivamente esperaríamos que la comunidad B tenga una diversidad que fuera exactamente el doble que la diversidad de la comunidad A. Usando la medida ${ }^{q} D$, efectivamente la comunidad B tendrá el doble de diversidad 
Cuadro 1. Estudios de caso de los cuales fueron obtenidos los datos para el cálculo de números efectivos en este trabajo*

\begin{tabular}{|c|c|c|c|c|c|}
\hline Estudio de caso* & Región geográfica & Grupo biológico & Condiciones comparadas & $\begin{array}{l}\text { Indice de } \\
\text { diversidad } \\
\text { empleado }\end{array}$ & $\begin{array}{c}\text { Comparación } \\
\text { estadística entre } \\
\text { índices }\end{array}$ \\
\hline $\begin{array}{l}\text { 1. Hongos de La } \\
\text { Laguna }\end{array}$ & $\begin{array}{l}\text { La Laguna, Coa- } \\
\text { huila }\end{array}$ & $\begin{array}{l}\text { Géneros de hon- } \\
\text { gos del suelo }\end{array}$ & $\begin{array}{l}\text { Tres predios en los mu- } \\
\text { nicipios de Matamoros y } \\
\text { Viesca }\end{array}$ & $\begin{array}{l}\text { Shannon y } \\
\text { Simpson }\end{array}$ & $\begin{array}{l}\text { Intervalos de con- } \\
\text { fianza al 95\% para } \\
\text { el índice de Shan- } \\
\text { non }\end{array}$ \\
\hline $\begin{array}{l}\text { 2. Moluscos de } \\
\text { El Edén }\end{array}$ & $\begin{array}{l}\text { Reserva ecológica } \\
\text { El Edén, Quintana } \\
\text { Roo }\end{array}$ & $\begin{array}{l}\text { Especies de } \\
\text { moluscos dul- } \\
\text { ceacuícolas }\end{array}$ & $\begin{array}{l}\text { Época de sequía y época de } \\
\text { lluvias en } 5 \text { sitios }\end{array}$ & Shannon & $\begin{array}{l}\text { Prueba pareada } \\
\text { de rangos de Wil- } \\
\text { coxon (Wilcoxon } \\
\text { Matched-Pairs } \\
\text { Signed Ranks test) }\end{array}$ \\
\hline $\begin{array}{l}\text { 3. Lagartijas del } \\
\text { Soconusco }\end{array}$ & $\begin{array}{l}\text { Soconusco, Chi- } \\
\text { apas }\end{array}$ & $\begin{array}{l}\text { Especies de lagar- } \\
\text { tijas }\end{array}$ & $\begin{array}{l}\text { Nueve tipos de hábitat, } \\
\text { incluyendo cafetales, } \\
\text { bosques primarios y un } \\
\text { pastizal }\end{array}$ & Shannon & $\begin{array}{l}\text { Prueba de } t \\
\text { modificada por } \\
\text { Hutcheson }\end{array}$ \\
\hline $\begin{array}{l}\text { 4. Murciélagos } \\
\text { del istmo }\end{array}$ & $\begin{array}{l}\text { Laguna Inferior, } \\
\text { istmo de Tehuante- } \\
\text { pec, Oaxaca }\end{array}$ & $\begin{array}{l}\text { Especies de } \\
\text { mamíferos (en este } \\
\text { trabajo se reanali- } \\
\text { zan sólo los datos } \\
\text { de murciélagos) }\end{array}$ & $\begin{array}{l}\text { Cuatro localidades con dife- } \\
\text { rentes tipos de vegetación }\end{array}$ & Shannon & $\begin{array}{l}\text { Análisis de vari- } \\
\text { anza (ANOVA) y } \\
\text { prueba de Tukey- } \\
\text { Kramer para la } \\
\text { diversidad prome- } \\
\text { dio, intervalos de } \\
\text { confianza al 95\% } \\
\text { para la diversidad } \\
\text { acumulada }\end{array}$ \\
\hline
\end{tabular}

*Referencias: estudio de caso 1, Samaniego-Gaxiola y Chew-Madinaveitia (2007); estudio de caso 2, Cózatl-Manzano y NaranjoGarcía (2007); estudio de caso 3, Macip-Ríos y Muñoz-Alonso (2008); estudio de caso 4, López et al. (2009).

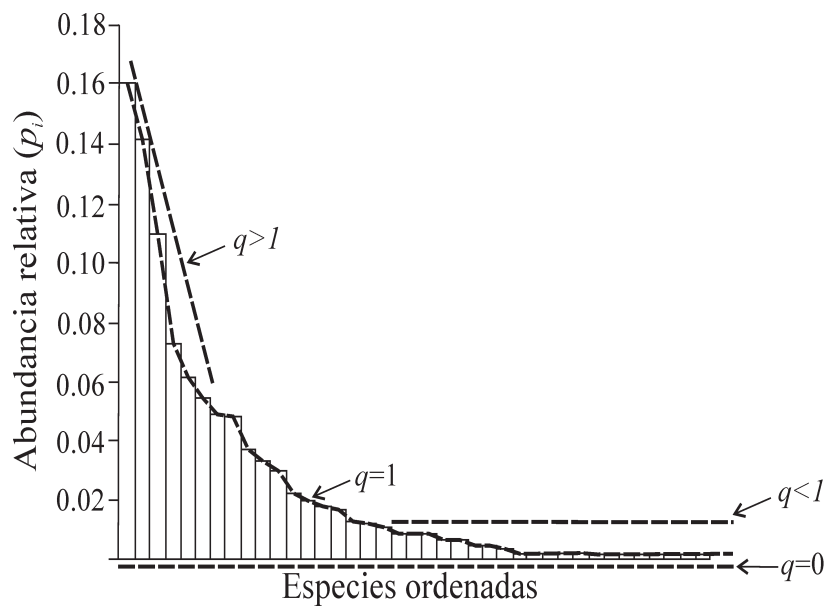

Figura 1. Distribución de las abundancias de las especies en una comunidad virtual. La más abundante a la izquierda; las más raras a la derecha. Líneas discontinuas: componentes de la comunidad considerados al evaluar la diversidad $\left({ }^{q} D\right)$ con distintos valores del parámetro $q$. En el caso más extremo $(\mathrm{q}=0)$, la diversidad es igual a la riqueza de especies, sin considerar las abundancias relativas; en otros casos la diversidad puede dar más peso a las especies raras $(q<1)$, a las dominantes $(q>1)$, o considerar a todas con un peso proporcional a su abundancia $(q=1)$. que la comunidad A (independientemente de los valores de $p_{i}$ y de $q$ ). Además, el principio de replicación (Jost, $2007,2010)$ dice que si tenemos $N$ comunidades con el mismo número de especies e igualmente diversas, sin ninguna especie compartida entre ellas, la diversidad del conjunto de estas comunidades debe ser igual a $N$ veces la diversidad de una sola comunidad. La diversidad medida con números efectivos se comporta de acuerdo con estas propiedades, mientras que con índices como el de Shannon o el de Simpson esto no ocurre (Jost, 2006, 2007). Además, se expresan en unidades que han sido llamadas especies igualmente comunes (MacArthur, 1965), número efectivo de especies (Hill, 1973; Jost, 2006; Tuomisto 2010a y b) o número de especies equivalentes (Jost , 2006; 2007). Por ejemplo, si para una determinada comunidad con 20 especies encontramos una diversidad con el índice de Shannon de 2.85 nats, al convertirlo en exp (H') tendríamos una diversidad una diversidad de ${ }^{1} D=17.29$ especies efectivas. Esto significa que la diversidad de esa comunidad es igual a la diversidad que tendría una comunidad virtual con 17.29 especies, en la cual todas las especies tuvieran exactamente la misma abundancia. Por lo tanto, la diversidad ${ }^{q} D$ expresada como un número efectivo de especies mide la 
diversidad que tendría una comunidad integrada por $i$ especies igualmente comunes $\left(p_{1}=p_{2}=p_{3}=\ldots p_{S}\right)$. El término efectivo se utiliza igualmente en física, economía, genética y otras disciplinas. Por ejemplo, en ciencias políticas se pueden comparar los sistemas electorales entre países mediante el uso del número efectivo de partidos políticos, que pondera el número de partidos con su correspondiente participación relativa en las votaciones.

Una ventaja de expresar la diversidad de una comunidad en números de especies efectivas es que permite comparar la magnitud de la diferencia en la diversidad de 2 o más comunidades. La mayoría de los estudios que utilizan el índice de Shannon como medida de diversidad suelen comparar la diversidad entre comunidades mediante pruebas como la $t$ modificada por Hutcheson, para comparar 2 índices de Shannon (Zar, 1999) o mediante intervalos de confianza al $95 \%$ obtenidos a través de técnicas de remuestreo (como bootstrap). Este tipo de pruebas estadísticas pueden ser útiles para concluir si hay diferencias significativas entre valores del índice de Shannon. Pero desde el punto de vista biológico, además de las diferencias estadísticamente significativas, resulta aún más interesante conocer la magnitud de la diferencia en la diversidad de 2 o más comunidades. Esto no es fácil de evaluar con el índice de entropía de Shannon pues su comportamiento no es lineal (véase explicación y pruebas matemáticas en Jost, 2006). En este trabajo, además de comparar estadísticamente la diversidad por medio de intervalos de confianza (obtenidos mediante estimadores, como se explica en la siguiente sección), se compara directamente qué tan diferente es la diversidad entre comunidades de la siguiente manera: si una comunidad A tiene una diversidad $D_{A}$ mayor que la diversidad $D_{B}$ de la comunidad B, entonces la comunidad A tendrá $D_{A} / D_{B}$ veces más diversidad que la comunidad B. O de otra manera, si la comunidad A tiene una diversidad $D_{A}$ mayor que la diversidad $D_{B}$ de la comunidad $\mathrm{B}$, entonces la comunidad $\mathrm{B}$ tendrá solamente un porcentaje $\left(D_{B} \times 100\right) / D_{A}$ de la diversidad que tiene la comunidad A.

Análisis de datos. Diversidad observada vs. diversidad estimada. Debido a que una comunidad generalmente está integrada por un gran número de especies con abundancias relativamente bajas, cuando se realiza un muestreo y cada individuo se reconoce taxonómicamente, algunas especies raras en la comunidad pueden no estar representadas en la muestra. Este es el caso más común en trabajos de campo de ecología de comunidades, porque el esfuerzo de muestreo invertido suele ser insuficiente para registrar a todas las especies por restricciones logísticas. El resultado es que la diversidad observada en la muestra suele ser menor a la diversidad que se esperaría encontrar en la comunidad. Para subsanar este problema se han generado modelos matemáticos que permiten estimar la diversidad total de la comunidad. Algunos ejemplos de tales modelos son los estimadores de Chao, Jackknife y ACE (Chao, 1984, 1987; Moreno, 2001; Espinosa-Escalante, 2003; Chao et al., 2009; Beck y Schwanghart, 2010) los cuales se han vuelto de uso común para estimar la riqueza de especies. Si en los estudios de caso reanalizados hubiera un submuestreo de las comunidades, entonces la diversidad observada pudiera ser distinta de la diversidad esperada. Por ello, en este trabajo se calculan los valores de números efectivos con 3 valores de $q(0,1$ y 2$)$ con base en la información observada en la muestra y, además, se utilizan estimadores de estos mismos parámetros. Los estimadores utilizados consideran como medida de completitud de los inventarios la cobertura de la muestra (sample coverage), que representa la fracción de las abundancias totales de la comunidad que están representadas en la muestra; es decir, el número de individuos del universo del cual se obtiene la muestra, dividido entre el número de individuos observados en la muestra (Chao y Shen, 2003). Una razón para utilizar la cobertura de la muestra es que la representatividad de una muestra depende no solo del número de especies que faltan, sino también de sus abundancias promedio. Una muestra que no logra registrar el $50 \%$ de las especies parece muy mala, pero si ese $50 \%$ faltante representa solo el $1 \%$ de los individuos totales, entonces tal vez la muestra pueda considerarse aceptable, aún cuando no detecte muchas especies. Teóricamente, es imposible estimar la riqueza a partir de muestras pequeñas; sin embargo, la riqueza de especies, a una cobertura de muestra dada, es un número bien definido que puede ser estimado con la precisión que se desee. El inverso en la cobertura de la muestra es la probabilidad de descubrir una nueva especie si se añade una observación adicional (un nuevo individuo). Es decir, entre más completa (o mejor cubierta) se tenga la muestra, es menos probable que un individuo colectado en el siguiente muestreo sea de una especie no registrada. Un estimador bien conocido para esta probabilidad es la proporción de especies con un solo individuo (singletons) en la muestra. En teoría, los estimadores basados en la cobertura de las muestras tienen una base sólida y funcionan muy bien en simulaciones (Chao y Shen, 2003). Para cada valor de $q$, se han propuesto distintos estimadores; para simplificar, en este trabajo, se seleccionó solamente uno de ellos para cada orden de diversidad. Para la diversidad de orden 0 (riqueza de especies), se utilizó el estimador no-paramétrico ACE (Abundance-based coverage estimator, Chao y Lee, 1992). Para la diversidad de orden 1 (exponencial del índice de Shannon), se utilizó un estimador del índice de Shannon propuesto para casos donde no se tiene un conocimiento completo de la comunidad (Bias-corrected Shannon diversity estimator, Chao y Shen, 2003). Este estimador corrige 
el sesgo del submuestreo y sus intervalos de confianza han demostrado tener una probabilidad de cobertura de muestra satisfactoria, por lo que funciona razonablemente bien aún cuando hay una fracción relativamente grande de especies faltantes (Chao y Shen, 2003; Beck y Schwanghart, 2010). Cuando se calcula el exponencial de este estimador, se obtiene una buena medida de diversidad estimada, recomendable porque no tiene sesgo cuando en la muestra se han registrado por lo menos la mitad de las especies de la comunidad (Beck y Schwanghart, 2010). Finalmente, para la diversidad de orden 2 (inverso del índice de Simpson) se utilizó el estimador MVUE (Minimum variance unbiased estimator, Chao y Shen, 2010). Todos estos estimadores fueron calculados para las condiciones de cada estudio de caso mediante el programa SPADE (Chao y Shen, 2010).

\section{Resultados}

Estudio de caso 1: hongos del suelo en La Laguna (Samaniego-Gaxiola y Chew-Madinaveitia, 2007). La riqueza $(q=0)$ claramente diferencia al predio de Tierra Blanca como el más rico en géneros de hongos (23 géneros), pero consideró los predios de San Jorge y El Chupón con la misma riqueza (12 géneros en cada predio, Fig. 2A; Cuadro 2). Al incluir todos los géneros y su abundancia relativa en la medida de diversidad $(q=1)$, se encontró la misma tendencia descrita por los autores, pues a pesar de tener la misma riqueza, el predio San Jorge es claramente más diverso que El Chupón (Fig. 2B). Finalmente, con la medida de diversidad de orden $2(q=2)$ el predio San Jorge alcanza una diversidad incluso mayor que la del predio Tierra Blanca (Fig. 2C) y se observó que en este predio los géneros comunes tienen mayor equidad que los géneros comunes de Tierra Blanca (Fig. 2D).

Con la medida de diversidad de orden 1 se encontró que el predio Tierra Blanca tiene una diversidad igual a la que tendría una comunidad teórica de 6.82 géneros donde todos ellos tuvieran la misma abundancia. Por su parte, el predio San Jorge tiene una diversidad igual a la que tendría una comunidad de 5.58 géneros efectivos, y El Chupón tiene 3.30 géneros efectivos. Al expresar estas equivalencias, se concluye que el predio Tierra Blanca es 1.22 veces más diverso en géneros de hongos que el predio San Jorge. Por su parte, el predio San Jorge es 1.69 veces más diverso que el predio El Chupón, y el predio Tierra Blanca tiene más del doble (2.07 veces) de la diversidad en géneros de El Chupón. Dicho de otra manera, El Chupón tiene apenas $48.34 \%$ de la diversidad que tiene Tierra Blanca.

Estudio de caso 2: moluscos de El Edén (Cózatl-Manzano y Naranjo-García, 2007). Con la diversidad de orden 1 (Fig. 3A) se observan tendencias muy similares a las publicadas por los autores con el índice de Shannon. El
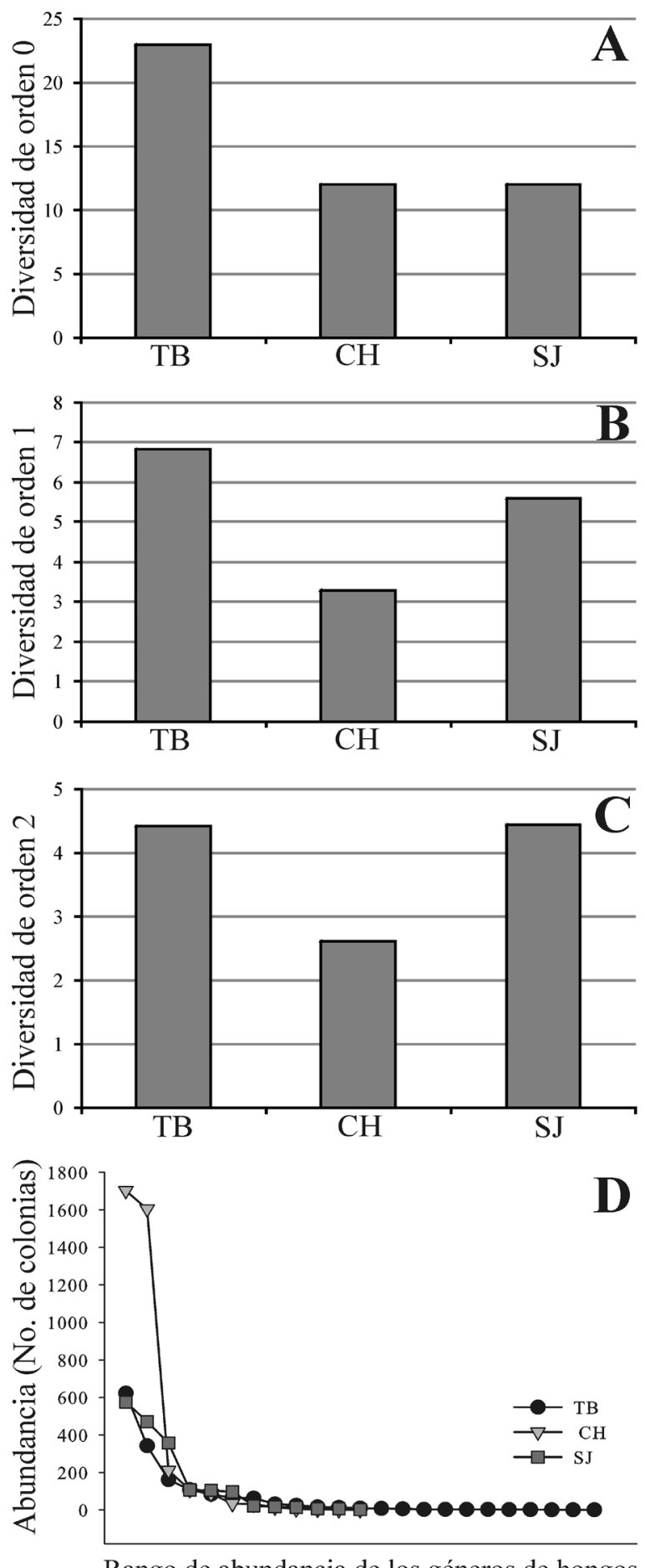

Rango de abundancia de los géneros de hongos

Figura 2. Diversidad de los géneros de hongos de suelo aislados de 3 predios (TB: Tierra Blanca, CH: El Chupón, y SJ: San Jorge) en La Laguna, Coahuila, por Samaniego-Gaxiola y ChewMadinaveitia (2007). A, riqueza de especies (diversidad de orden $q=0$ ); B, diversidad de orden $q=1$; C, diversidad de orden $q=2$; $\mathrm{D}$, gráfica de rango-abundancia de los hongos en los 3 predios. 
Cuadro 2. Resultados del análisis de la diversidad de 4 estudios de caso. Se indican los valores del índice de Shannon originalmente publicados y los valores de números efectivos observados y estimados

\begin{tabular}{|c|c|c|c|c|c|c|c|c|}
\hline \multirow[t]{2}{*}{ Estudio de caso } & \multirow{2}{*}{$\begin{array}{l}\text { Condiciones } \\
\text { comparadas }\end{array}$} & \multirow{2}{*}{$\begin{array}{l}\text { Índice de } \\
\text { Shannon }\end{array}$} & \multicolumn{3}{|c|}{ Diversidad observada } & \multicolumn{3}{|c|}{ Diversidad estimada } \\
\hline & & & ${ }^{0} D$ & ${ }^{1} D$ & ${ }^{2} D$ & ${ }^{o} D$ & ${ }^{1} D$ & ${ }^{2} D$ \\
\hline \multirow{3}{*}{$\begin{array}{l}\text { 1. Hongos de la } \\
\text { Laguna }\end{array}$} & Tierra Blanca & 1.89 & 23 & 6.82 & 4.42 & 25.20 & 6.90 & 4.43 \\
\hline & El Chupón & 1.19 & 12 & 3.30 & 2.61 & 14.10 & 3.31 & 2.61 \\
\hline & San Jorge & 1.72 & 12 & 5.58 & 4.44 & 12.00 & 5.59 & 4.45 \\
\hline \multirow{10}{*}{$\begin{array}{l}\text { 2. Moluscos de El } \\
\text { Edén* }\end{array}$} & $1 \mathrm{D}$ & 1.57 & 3 & 2.98 & 2.96 & 3.00 & 3.00 & 3.55 \\
\hline & $1 \mathrm{R}$ & 3.02 & 9 & 8.12 & 7.76 & 9.20 & 8.32 & 8.69 \\
\hline & $2 \mathrm{D}$ & 0.92 & 2 & 1.89 & 1.80 & 3.00 & 2.90 & 3.00 \\
\hline & $2 \mathrm{R}$ & 0.00 & 1 & 1.00 & 1.00 & 1.00 & 1.00 & 1.00 \\
\hline & $3 \mathrm{D}$ & 2.58 & 7 & 6.00 & 5.44 & 8.00 & 7.04 & 7.00 \\
\hline & $3 \mathrm{R}$ & 2.74 & 9 & 6.67 & 5.74 & 10.20 & 7.06 & 6.17 \\
\hline & $4 \mathrm{D}$ & 2.24 & 5 & 4.71 & 4.45 & 8.80 & 9.00 & 10.50 \\
\hline & $4 \mathrm{R}$ & 2.85 & 8 & 7.19 & 6.67 & 8.90 & 8.81 & 9.50 \\
\hline & $5 \mathrm{D}$ & 1.82 & 5 & 3.54 & 2.92 & 5.00 & 3.83 & 3.13 \\
\hline & $5 \mathrm{R}$ & 2.87 & 10 & 7.33 & 5.72 & 14.80 & 9.43 & 6.88 \\
\hline \multirow{9}{*}{$\begin{array}{l}\text { 3. Lagartijas del } \\
\text { Soconusco** }^{* *}\end{array}$} & Cdiv. I & 1.61 & 8 & 5.01 & 3.93 & 8.50 & 5.30 & 4.10 \\
\hline & Cdiv. II & 1.57 & 7 & 4.82 & 3.60 & 7.00 & 4.89 & 3.71 \\
\hline & SM & 1.55 & 7 & 4.70 & 3.35 & 7.50 & 5.48 & 3.67 \\
\hline & $\mathrm{P}$ & 1.42 & 5 & 4.12 & 3.60 & 5.00 & 4.20 & 3.85 \\
\hline & Cdiv.V & 1.12 & 5 & 3.07 & 2.62 & 8.90 & 3.41 & 2.72 \\
\hline & Cdiv. III & 1.07 & 7 & 2.91 & 1.89 & 7.40 & 3.16 & 1.92 \\
\hline & C.I. micheliana & 1.06 & 4 & 2.89 & 2.55 & 4.00 & 2.98 & 2.64 \\
\hline & $\mathrm{BM}$ & 1.05 & 4 & 2.87 & 2.39 & 6.40 & 3.61 & 2.67 \\
\hline & Cdiv. IV & 0.83 & 5 & 2.30 & 1.64 & 5.40 & 2.45 & 1.67 \\
\hline \multirow[t]{4}{*}{$\begin{array}{l}\text { 4. Murciélagos del } \\
\text { Istmo }\end{array}$} & $\begin{array}{l}\text { Montecillo Santa } \\
\text { Cruz } 1\end{array}$ & 2.00 & 13 & 7.41 & 5.12 & 14.00 & 7.64 & 5.23 \\
\hline & $\begin{array}{l}\text { Montecillo Santa } \\
\text { Cruz } 2\end{array}$ & 1.63 & 11 & 5.24 & 4.14 & 16.50 & 5.63 & 4.22 \\
\hline & Las Palmas & 2.23 & 16 & 9.58 & 8.16 & 21.00 & 9.90 & 8.35 \\
\hline & Huamuchil & 1.78 & 10 & 6.16 & 5.21 & 16.30 & 6.71 & 5.46 \\
\hline
\end{tabular}

Diversidad observada y estimada: ${ }^{0} \mathrm{D}$ (riqueza de especies), ${ }^{1} \mathrm{D}$ y ${ }^{2} \mathrm{D}$ (denotan medidas de diversidad de orden 0,1 y 2 , respectivamente).

* Cinco sitios en la época de sequía (D) y en la época de lluvias (R).

** Cinco cafetales diversificados (Cdiv. I, Cdiv. II, Cdiv. III, Cdiv. IV y Cdiv.V), cafetal con Inga micheliana (C.I. micheliana), selva mediana (SM), pastizal (P) y bosque mesófilo (BM).

mayor contraste en la diversidad de moluscos entre épocas se detecta en el sitio 1, en el cual durante la época de sequía la diversidad es de 2.98 especies efectivas, mientras que durante la época de lluvias la diversidad es de 8.12 especies efectivas (Fig. 3A). Es decir, durante la época de lluvias la diversidad de moluscos es 2.72 veces mayor que durante la sequía. Esta magnitud de la diferencia entre épocas también se puede expresar diciendo que en la sequía se reduce $63.15 \%$ de la diversidad presente durante la época de lluvias, lo cual es una diferencia muy importante. En el sitio 5 la diferencia en la magnitud de la diversidad es más del doble durante la época de lluvias, mientras que en los otros sitios las diferencias no son tan marcadas. Al utilizar un estimador de la diversidad se observan cambios en los sitios 3 y 4 , donde la tendencia se revierte (Fig. 3B): en el sitio 3 la diversidad resultó igual en ambas épocas, 
y en el sitio 4 la diversidad fue incluso mayor durante la época de sequía, aunque estas diferencias no fueron estadísticamente significativas considerando los intervalos de confianza al 95\% (Fig. 3B).

Considerando solamente la riqueza de especies observada y la riqueza esperada de acuerdo con el estimador ACE (riqueza de especies ${ }^{\circ} D$, Cuadro 2), se pudo calcular que tan completos son los inventarios de los sitios para representar la máxima riqueza potencial de acuerdo con el muestreo. El sitio 4 durante la época de sequía tuvo la menor completitud del inventario de especies (57\%) (Fig. 3A), por lo que la diferencia entre la diversidad observada y la estimada en ese sitio fue muy distinta, y el intervalo de confianza al 95\% para la diversidad esperada de esta comunidad es muy amplio. En el sitio 2 en sequía y en el sitio 5 en lluvias los intervalos de confianza para la diversidad estimada son también muy amplios debido a que tienen completitud de 67 y $68 \%$, respectivamente.

Estudio de caso 3: lagartijas del Soconusco (MacipRíos y Muñoz-Alonso, 2008). La diversidad observada de orden 1 mostró la misma tendencia de variación entre sitios que la encontrada usando el índice de Shannon (Fig. 4A; Cuadro 2). Con esta medida de diversidad se puede decir que las comunidades de lagartijas de esta región del Soconusco chiapaneco tienen una diversidad máxima de 5 especies efectivas. Resulta de gran interés el incremento en la diversidad de orden 1 que se obtiene en los bosques primarios (selva mediana y bosque mesófilo) al utilizar un modelo de estimación (Fig. 4B). Con la diversidad observada de orden 1 la selva mediana ocupaba el tercer lugar con mayor diversidad, y el bosque mesófilo el octavo entre los 9 sitios estudiados (Fig. 4A). Sin embargo, con la diversidad estimada la selva mediana tiene el primer lugar de diversidad y el bosque mesófilo el quinto (Fig. 4B), lo cual remarca la importancia de la conservación de los bosques primarios para este grupo de reptiles.

Además, para complementar una visión acerca de la relación entre la altitud y la diversidad de lagartijas, podemos comparar estos bosques primarios con vegetación modificada ubicada a la misma altitud utilizando los valores de diversidad estimada de orden 1. La selva mediana, con 5.48 especies efectivas, tuvo 2.23 veces más diversidad que el cafetal diversificado IV (con 2.45 especies efectivas), aún cuando ambos sitios están ubicados a $700 \mathrm{~m}$ de altitud. Por los intervalos de confianza al 95\% se puede decir que la diversidad de la selva mediana es estadísticamente mayor que la diversidad tanto del cafetal diversificado IV como del cafetal de Inga micheliana (Fig. 4B). Por su parte, la diversidad del bosque mesófilo (3.61 especies efectivas) fue ligeramente mayor que la diversidad del cafetal diversificado V (3.41 especies efectivas) que está ubicado a una altitud similar, aunque en este caso la diferencia no fue estadísticamente significativa.

Estudio de caso 4: murciélagos del istmo (López et al., 2009). Los valores de diversidad estimada de orden 1 coinciden con lo descrito por López et al. (2009) (Fig. 5B; Cuadro 2), aunque con el modelo de estimación utilizado en este reanálisis los intervalos de confianza son mucho más amplios y se puede concluir que no hay diferencias estadísticamente significativas entre sitios. La mayor diversidad de Las Palmas equivale a la diversidad que tendría una comunidad de murciélagos con 9.58 especies efectivas. Esta diversidad es 1.83 veces mayor que la diversidad del sitio menos diverso (Montecillo Santa Cruz 2, con 5.24 especies efectivas). Es decir, entre el sitio de Las Palmas (donde predomina el bosque espinoso mixto, la vegetación riparia, el pastizal y los cultivos) y el sitio de Montecillo Santa Cruz 2 (donde predominan los cultivos, el pastizal y la vegetación riparia), hay una diferencia de
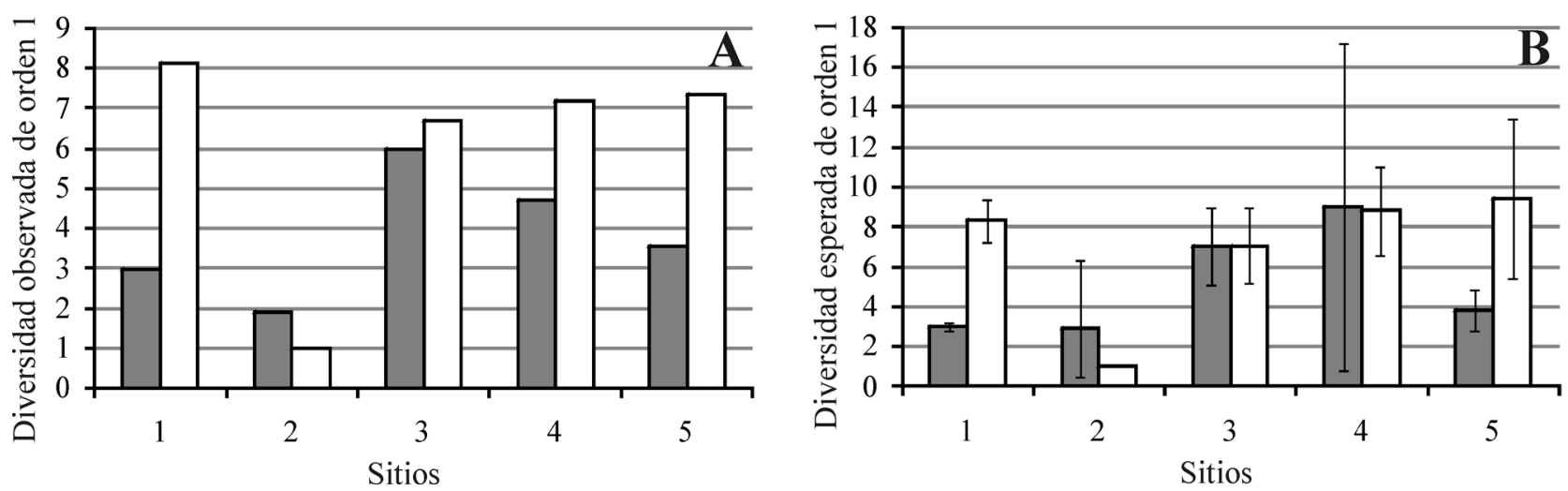

Figura 3. Diversidad $\left({ }^{1} D\right)$ observada (A) y esperada (B) de moluscos dulceacuícolas registrados por Cózatl-Manzano y Naranjo-García (2007) en 5 sitios de la Reserva Ecológica El Edén, Quintana Roo, durante la época de sequía (barra gris) y la época de lluvias (barra blanca). Las barras de error en B son intervalos de confianza al 95\%. 
$43.13 \%$ en la diversidad. Este resultado contrasta con la interpretación a la que se puede llegar cuando únicamente se consideran los valores del índice de Shannon, ya que la diferencia en diversidad de murciélagos entre ambos sitios es apenas de $26.90 \%$.

Al utilizar como medida la diversidad estimada de orden 2 , todos los sitios resultan con un menor número de especies efectivas, pues esta medida se centra solamente en las especies más abundantes (Fig. 5C). Con la diversidad de orden 2, el sitio de Huamuchil se posiciona como el segundo sitio más diverso (Fig. 5C) y no en el tercer lugar, como con la diversidad de orden 1 (Fig. 5A), lo que se debe a que en Huamuchil la especie más abundante (Phyllostomus discolor) tiene menos de la mitad del número de individuos que la más abundante en cualquiera de los otros sitios, por lo que hay una mayor equidad en la distribución de abundancias entre las especies comunes (Fig. 5D).

\section{Discusión}

El uso de números efectivos permitió una mejor interpretación de la diversidad de las comunidades y las comparaciones entre sitios. Los datos del estudio de caso 1 (hongos del suelo en La Laguna) han sido especialmente útiles para ejemplificar el significado del exponente $q$ de las medidas de diversidad, para comparar la magnitud de la diferencia en diversidad entre las comunidades estudiadas y para demostrar el uso de medidas de diversidad alfa a una resolución taxonómica distinta a la de especie. Este último punto es importante de resaltar debido a que el concepto de diversidad se puede aplicar para medir distintas clases de diversidad, dependiendo del nivel de clasificación que se haga de las unidades de observación (Tuomisto, 2010b). En el estudio de caso 1, las unidades de observación en las comunidades son los géneros, no las especies. SamaniegoGaxiola y Chew-Madinaveitia (2007) encontraron que la diversidad de géneros de hongos, medida con el índice de entropía de Shannon, fue estadísticamente distinta (los intervalos de confianza no se traslapan) entre los 3 predios agrícolas comparados, siendo mayor en el predio Tierra Blanca, intermedia en el predio San Jorge y baja en el predio El Chupón. Estas diferencias se corroboraron en el reanálisis de este trabajo con la diversidad de orden 1 (Fig. 2B). Lo que las medidas de los números efectivos aportan, y que no es posible visualizar con el índice de Shannon, es la magnitud de las diferencias en diversidad entre los 3 predios y los elementos que determinan dichas diferencias. La baja diversidad en El Chupón se debe a que en ese predio se encontraron 2 géneros (Penicillium y Fusarium) altamente dominantes (Fig. 2D), que representan $87 \%$ de las colonias detectadas. Por otro lado, la alta diversidad de hongos en el predio San Jorge $(q=2)$ se debe a que esta medida no considera en absoluto los géneros con baja abundancia, muchos de ellos encontrados en Tierra Blanca (Fig. 2D) y se enfocó en la abundancia relativa de los géneros más comunes. Además, la diferencia en diversidad entre El Chupón y Tierra Blanca sugiere que en ambos sitios hay algunos factores que han provocado la disminución en la diversidad de géneros de hongos en 51.66\%. Al comparar con el índice de entropía de Shannon, la diferencia entre estos 2 últimos predios fue de 0.698 bits, lo cual indicaría que Tierra Blanca es apenas 1.58 veces más diverso que El Chupón.

Para la diversidad de moluscos dulceacuícolas en 5 sitios de El Edén, Cózatl-Manzano y Naranjo-García
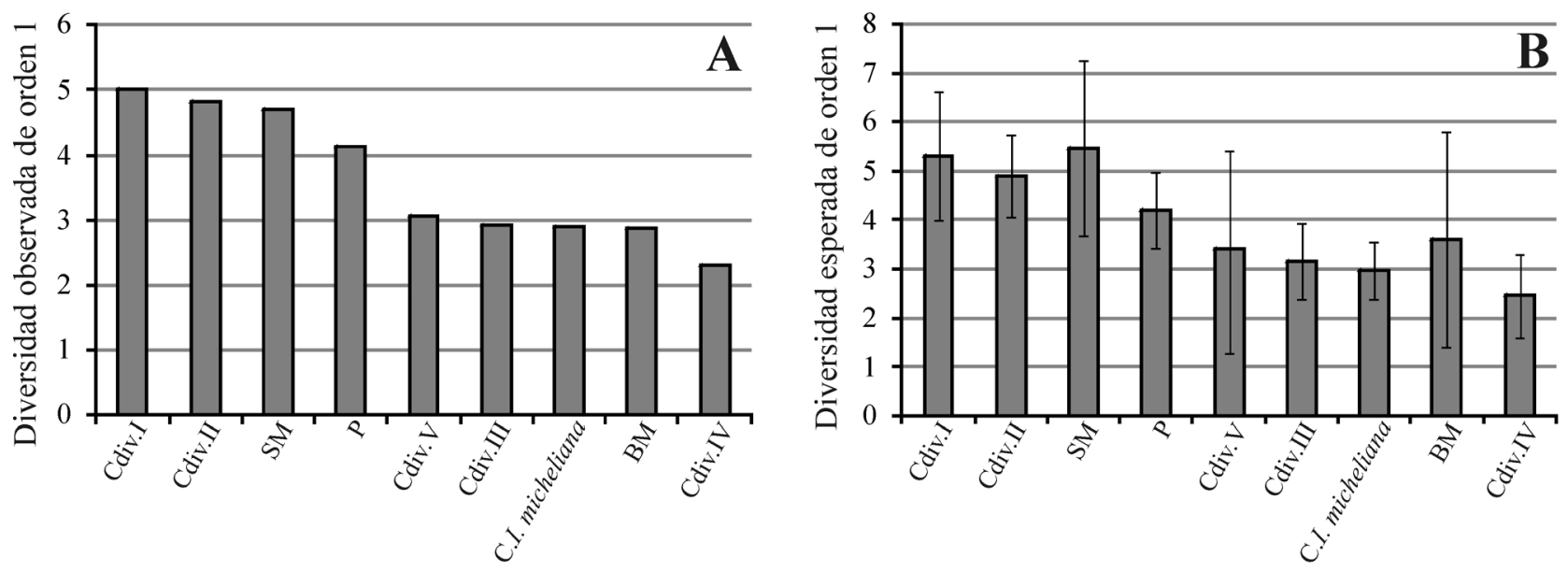

Figura 4. Diversidad $\left({ }^{I} D\right)$ observada (A) y esperada (B) de lagartijas registradas por Macip-Ríos y Muñoz-Alonso (2008) en 9 sitos de la región del Soconusco, Chiapas, incluyendo 5 sitios de cafetal diversificado (Cdiv. I al V), un sitio de cafetal con sombra monoespecífica de Inga micheliana (C.I. micheliana), un pastizal inducido (P), un sitio de selva mediana (SM), y un sitio de bosque mesófilo (BM). Las barras de error en B son intervalos de confianza al 95\%. 

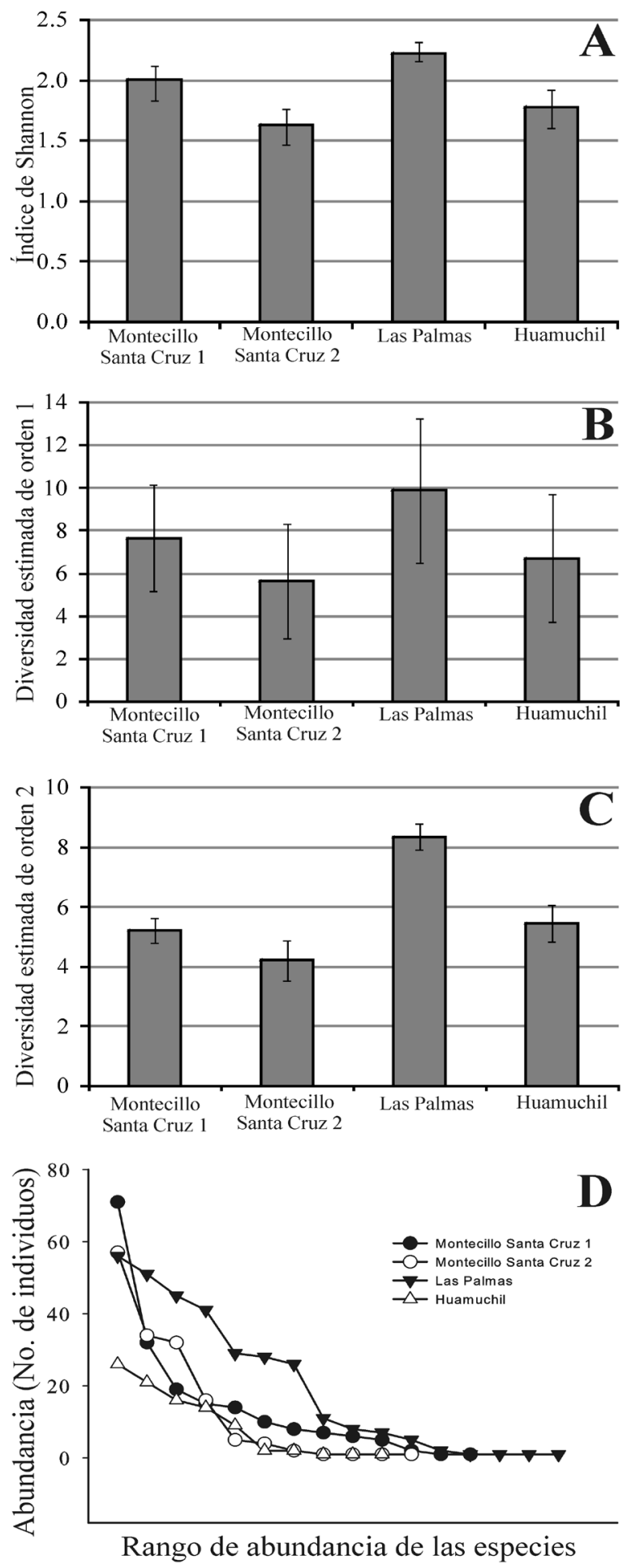

Figura 5. Índice de entropía de Shannon (A), diversidad estimada (B: ${ }^{l} D, \mathrm{C}:{ }^{2} D$ ) y patrón de rango-abundancia (D) de los murciélagos capturados con redes de niebla por López et al. (2009) en 4 sitios del istmo de Tehuantepec, Oaxaca. Las barras de error en A, B y C son intervalos de confianza al 95\%.
(2007), utilizando el índice de Shannon con logaritmo base 2 , no encontraron diferencias estadísticamente significativas entre la época de sequía y la época de lluvias por lo que concluyen que el índice de Shannon "dio poca información”. Sin embargo, sólo uno de los sitios (sitio 2) tuvo menor diversidad ( 1 especie) durante la época de lluvias, mientras que en los otros 4 sitios, la diversidad durante esta época fue mayor (Cuadro 2).

Los autores consideraron que para estudios posteriores es recomendable realizar un esfuerzo de muestreo durante más tiempo y a intervalos más frecuentes, combinando técnicas de muestreo. Por lo anterior, utilizando la información del estudio de caso 2 , en este trabajo se ha comparado la diversidad observada en las muestras con la diversidad estimada mediante modelos basados en el supuesto de que las muestras no incluyen todas las especies que existen en la comunidad. La razón de las diferencias encontradas entre los sitios radica, al parecer, en un nivel de muestreo insuficiente. Cózatl-Manzano y Naranjo-García (2007) utilizaron diferente número de cuadros de muestreo en los 5 sitios, muestreando con mayor intensidad en sitios de vegetación densa y con menor intensidad en lagunas con pocas asociaciones vegetales y substratos, pero no proporcionan información más precisa sobre su esfuerzo de muestreo. En general, si una comunidad está submuestreada, los estimadores de diversidad basados en la cobertura de la muestra darán valores notablemente más altos que los de la diversidad observada (Chao y Shen, 2003).

Macip-Ríos y Muñoz-Alonso (2008) estudiaron la comunidad de lagartijas en 9 sitios a distinta altitud y con distinto tipo de vegetación y, con base en el índice de Shannon, concluyeron que hay diferencias significativas en la diversidad de lagartijas entre 2 grupos de sitios: uno con alta diversidad, integrado por la selva mediana, el pastizal y los cafetales diversificados I y II (ubicados a baja altitud), y otro con baja diversidad, conformado por los cafetales diversificados III, IV y V (ubicados a mediana altitud), el cafetal de Inga micheliana y el bosque mesófilo. Además, encontraron una relación inversa significativa entre la diversidad y la altitud. En general, los autores concluyeron que sus análisis de la diversidad de lagartijas apoyan la hipótesis de que los cafetales con sombra diversificada ubicados a menos de $700 \mathrm{~m}$ de altitud funcionan como un refugio para la biodiversidad, por lo que se requieren estrategias adecuadas para su conservación. Sin embargo, mencionan que sus inventarios tienen una completitud baja en la selva mediana y en el bosque mesófilo $(46.94 \%$ y $64.10 \%$ de acuerdo con el estimador ICE, respectivamente). En general, los resultados encontrados en este trabajo corroboran la observación de Macip-Ríos y Muñoz-Alonso (2008) en relación con la alta diversidad de lagartijas en cafetales bajo los $700 \mathrm{~m}$ de altitud con sombra 
diversificada. Sin embargo, el uso de medidas de números efectivos de especies y de estimadores de la diversidad permitió resaltar la importancia de la conservación de los bosques primarios, especialmente de la selva mediana, pues este hábitat tiene más del doble de la diversidad de lagartijas que un cafetal diversificado ubicado a la misma altitud.

Finalmente, López et al. (2009) encontraron diferencias significativas en la diversidad promedio de murciélagos por noche en 4 localidades del istmo de Tehuantepec, utilizando el índice de Shannon. El sitio Las Palmas fue significativamente más diverso que el sitio Huamuchil, y la diversidad acumulada con el índice de Shannon fue también significativamente más alta en Las Palmas que en los otros 3 sitios (Fig. 5A). Con las medidas utilizadas en este trabajo, más allá de la significancia estadística de las diferencias, se resalta cuánta diversidad se gana o se pierde entre los sitios. Además, se describen los elementos de la estructura de las comunidades que generan las diferencias en la diversidad de especies.

Ventajas de los números efectivos de especies como alternativa para evaluar la diversidad ecológica. La confusión en torno al concepto de diversidad de especies se debe en gran parte al uso y abuso de índices que reflejan la complejidad de composición (compositional complexity sensu Jost, 2010), como la entropía, que miden conceptos relacionados con la diversidad, pero no la diversidad misma. Una de las críticas más frecuentes sobre el uso de medidas como el índice de entropía de Shannon es que no tienen una interpretación biológica clara (Molinari, 1989; Ricotta, 2005), por ello, en muchas ocasiones se decide analizar la diversidad de las comunidades considerando únicamente la riqueza de especies, aunque se tengan datos sobre su distribución de abundancias. En este trabajo se ha ejemplificado el uso de números efectivos de especies que permiten incorporar los datos de abundancia y cumplen con una serie de propiedades matemáticas acordes con la interpretación intuitiva del concepto biológico de diversidad (Jost, 2006), y que evitan conclusiones potencialmente erróneas en biología de la conservación (Jost, 2009).

El concepto de diversidad está ligado al concepto de equidad (evenness). Para un determinado número de especies, la diversidad tiene un valor mínimo cuando la abundancia está concentrada solamente en una especie mientras que todas las demás quedan con un solo individuo, y tiene un valor máximo cuando todas las especies son igualmente comunes. La abundancia (medida como número de individuos, biomasa, o cualquier otra unidad) es, sin lugar a dudas, un parámetro ligado a la riqueza de especies. En la cotidianidad es necesario estar conscientes de que, al realizar inventarios biológicos, frecuentemente hay sesgos en el muestreo: al tomar una muestra de una comunidad con alta equidad es más probable registrar una mayor riqueza de especies que en una muestra tomada de una comunidad con muchas especies raras, aún cuando en realidad ambas comunidades tengan la misma riqueza de especies. Pero, ¿cuál es la relación matemática entre la riqueza, la diversidad y la equidad? Una aplicación reciente de los números efectivos de especies es la división del concepto de riqueza en 2 componentes independientes: la diversidad y la equidad (Jost, 2010). Estas transformaciones dan por resultado un conjunto de medidas con sentido biológico que pueden resultar útiles para elaborar un concepto unificado de la equidad (Jost, 2010).

En los estudios de caso que se analizaron se ve cómo la diversidad de distintos órdenes $(q=0, q=1, q=2)$ depende de ciertos componentes de la comunidad. Por ejemplo, si en la comunidad hay unas cuantas especies muy abundantes, la medida ${ }^{2} D$ se basará solamente en ellas, y no tomará en cuenta las especies raras. Por su parte, la medida ${ }^{1} D$ incluye tanto las especies comunes como las dominantes e incluso las raras (Fig. 1), y su valor será siempre mayor o por lo menos igual al valor de ${ }^{2} D$. Estas diferencias en los distintos órdenes de la diversidad permiten utilizar diferentes medidas para explorar la influencia de los procesos ecológicos y evolutivos en la abundancia de las especies coexistentes. Algunos procesos ecológicos (tales como las tasas de nacimiento, mortalidad, inmigración y emigración) intervienen en las abundancias de las especies coexistentes y actúan bajo la influencia de distintos factores bióticos y abióticos (Begon et al., 2006). Por otro lado, los patrones de abundancia relativa han sido moldeados por procesos evolutivos de ocupación de nichos en un paisaje adaptativo (Tokeshi, 1999); por lo tanto, los patrones de diversidad que consideran distintos elementos de la distribución de abundancias de una comunidad (Fig. 1) reflejan una combinación de fuerzas histórico-evolutivas y contemporáneo-ecológicas, que actúan como determinantes de la diversidad en las comunidades ecológicas (Tokeshi, 1999).

Los autores de los 4 estudios de caso analizaron las diferencias estadísticas del índice de Shannon entre las condiciones comparadas en cada caso. Sin embargo, las diferencias significativas no necesariamente tienen relación con la magnitud real de las diferencias, o con el significado biológico del efecto que tienen las condiciones comparadas en la diversidad. En biología de la conservación puede ser importante saber si la diversidad de un sitio prístino es significativamente distinta de la diversidad de un sitio perturbado en un sentido estadístico. Pero desde luego resulta mucho más interesante saber ¿qué tanta biodiversidad se pierde o se gana como resultado de la perturbación? Se puede expresar esta magnitud como proporciones o porcentajes de diferencia, 
según se ha ejemplificado en cada uno de los estudios de caso, lo cual podría sugerir ganancia o pérdida de diversidad. Resulta entonces de gran importancia seleccionar las medidas apropiadas para estudiar y entender la diversidad, particularmente cuando los valores obtenidos son un referente para proponer acciones de manejo de recursos o establecer estrategias de conservación biológica (Jost et al., 2010).

Perspectivas. Otra aplicación importante de las medidas de diversidad expresadas en números efectivos de especies es que constituyen una herramienta matemática poderosa para analizar la partición de la diversidad gamma en sus componentes alfa y beta. Si la medición de la diversidad alfa ha sido sumamente compleja, la base conceptual y metodológica del componente beta lo es aún más (Tuomisto, 2010). La propuesta de Jost (2007) de usar números efectivos en la partición de la diversidad gamma "constituye probablemente el aporte teórico más importante en la medición de la diversidad desde que Whittaker introdujo el concepto de diversidad beta en Ecología" (Ellison, 2010). Esta propuesta se basa en el teorema de la partición (Jost, 2007; 2010): si una medida de diversidad consiste de 2 componentes independientes, entonces dicha medida debe ser igual al resultado del primer componente multiplicado por el segundo componente. Siguiendo esta propuesta, cuando tenemos $N$ comunidades con igual peso, la diversidad beta es el número efectivo de comunidades distintas en la región, que varían de 1 hasta $N$ (Jost, 2006). Es decir, con la diversidad disponible que hay en toda la región, y considerando la diversidad alfa promedio: ¿cuántas comunidades virtuales podríamos tener que fueran completamente distintas en su composición de especies? Ese es el número efectivo o número equivalente de comunidades (sensu Jost, 2007) o número de unidades de composición (compositional units sensu Tuomisto, 2010).

La partición multiplicativa de la diversidad gamma en sus componentes alfa y beta, expresados como números efectivos, cumple con una serie de propiedades importantes que de otra manera (como en el caso de la partición aditiva del índice de Shannon) no se cumplen. Las diversidades alfa y beta deben ser independientes y nunca mayores que la diversidad gamma; alfa debe ser un promedio de la diversidad de las comunidades que integran la región; gamma debe estar completamente determinada por alfa y beta; y un valor dado debe referirse a la misma cantidad de diversidad (ya sea que venga del componente alfa, beta o gamma), de manera que los componentes puedan ser directamente comparados (Jost, 2007). Contar con métodos que cumplan estas propiedades es un punto crucial en el análisis de la diversidad regional, pues las recomendaciones que se deriven de dicho análisis podrían ayudar a la conservación efectiva de dicha diversidad (Jost et al., 2010).

Finalmente, vale la pena resaltar la posibilidad que ofrece el uso de números efectivos para evaluar otras facetas del concepto amplio de la biodiversidad. Todas las medidas descritas en este trabajo son neutrales, en el sentido de que se basan en el supuesto de que todas las especies de una comunidad son iguales. Sin embargo, recientemente Chao et al. (2010) derivaron un conjunto de medidas de diversidad no-neutrales que toman en cuenta tanto las abundancias de las especies como sus relaciones filogenéticas, para evaluar la diversidad evolutiva de las comunidades. Así, es posible cuantificar el número efectivo de linajes distintos en un intervalo de tiempo. De forma análoga se puede evaluar también, mediante el uso de números efectivos, la diversidad funcional o la diversidad genética, entre otras facetas de la biodiversidad.

En general, las tendencias que se detectan con la diversidad de orden 1 concuerdan con las tendencias originalmente detectadas por los autores de cada estudio de caso utilizando el índice de entropía de Shannon. Sin embargo, el uso de números efectivos de especies puede ayudar a mejorar los análisis de diversidad alfa por las siguientes razones: 1) aportan mayor robustez matemática, explicada con detalle en Jost (2006); 2) la concordancia de estas medidas con lo que intuitivamente se espera de una medida de diversidad; 3) la posibilidad de integrar diferentes componentes de la comunidad en función de la abundancia relativa, al modificar el valor del parámetro $q$; 4) la sencillez de expresar la diversidad en números efectivos y 5) la posibilidad de hacer comparaciones directas de cuánta diversidad se gana o se pierde de una comunidad a otra, independientemente de que haya diferencias estadísticamente significativas.

Muchos de los estudios empíricos que evalúan los componentes alfa, beta y gamma de la diversidad de especies, buscan proveer la base científica sobre la cual se apoyen las decisiones de manejo y conservación de la biodiversidad. Por ejemplo, los estudios pueden llegar a sugerir cómo priorizar los recursos disponibles (económicos o humanos) entre los sitios, localidades o hábitats de una región o de un área protegida. Para evitar decisiones irreversibles, es crucial invertir parte del esfuerzo en la búsqueda y revisión de los métodos de análisis más apropiados para cada caso.

\section{Agradecimientos}

Claudia E. Moreno agradece los consejos de Anne Chao para la selección de estimadores de diversidad y sus 
intervalos de confianza, así como las exhortaciones, recomendaciones y comentarios que amablemente hizo Lou Jost sobre este documento. Agradecemos a Bianca Delfosse la revisión del Abstract. Este trabajo es un resultado de los proyectos 95828 del Fondo Mixto CONACYTHIDALGO y 84127 del Fondo Sectorial de Ciencia Básica SEP-CONACYT.

\section{Literatura citada}

Beck, J. y W. Schwanghart. 2010. Comparing measures of species diversity from incomplete inventories: an update. Methods in Ecology \& Evolution 1:38-44.

Begon, M., C. R. Townsend y J. L. Harper. 2006. Ecology: from individuals to ecosystems, cuarta edición. Blackwell, Oxford. $738 \mathrm{p}$.

Chao, A. 1984. Nonparametric estimation of the number of classes in a population. Scandinavian Journal of Statistics 11:265-270.

Chao, A. 1987. Estimating the population size for capture-recapture data with unequal catchability. Biometrics 43:783-791.

Chao, A. y S. M. Lee. 1992. Estimating the number of classes via sample coverage. Journal of the American Statistical Association 87:210-217.

Chao, A. y T. J. Shen. 2003. Nonparametric estimation of Shannon's index of diversity when there are unseen species in sample. Environmental and Ecological Statistics 10:429-433.

Chao, A. y T. J. Shen. 2010. Program SPADE (Species prediction and diversity estimation). http://chao.stat.nthu.edu.tw; última consulta: 07.VII.2010.

Chao, A., R. Colwell, C. W. Lin y N. Gotelli. 2009. Sufficient sampling for asymptotic minimum species richness estimators. Ecology 90:1125-1133.

Chao, A., C. H. Chiu y L. Jost. 2010. Phylogenetic diversity measures based on Hill numbers. Philosophical Transactions of the Royal Society B 365:3599-3609.

Colwell, R. K. 2009. EstimateS: Statistical estimation of species richness and shared species from samples. Versión 8.2. http:// purl.oclc.org/estimates; última consulta: 05.V.2010.

Cózatl-Manzano, R. y E. Naranjo-García. 2007. First records of freshwater molluscs from the ecological reserve El Edén, Quintana Roo, Mexico. Revista Mexicana de Biodiversidad 78:303-310

Ellison, A. M. 2010. Partitioning diversity. Ecology 91:1962-1963.

Espinosa-Escalante, T. 2003. ¿Cuántas especies hay? Los estimadores no paramétricos de Chao. Elementos: ciencia y cultura 52:53-56.

Hill, M. O. 1973. Diversity and evenness: a unifying notation and its consequences. Ecology 54:427-432.

Hurlbert, S. H. 1971. The nonconcept of species diversity: a critique and alternative parameters. Ecology 52:577-586.

Jost, L. 2006. Entropy and diversity. Oikos 113:363-375.
Jost, L. 2007. Partitioning diversity into independent alpha and beta components. Ecology 88:2427-2439.

Jost, L. 2010. The relation between evenness and diversity. Diversity 2:207-232.

Jost, L., P. DeVries, T. Walla, H. Greeney, A. Chao y C. Ricotta. 2010. Partitioning diversity for conservation analyses. Diversity and Distributions 16:65-76.

López, J. A., C. Lorenzo, F. Barragán y J. Bolaños. 2009. Mamíferos terrestres de la zona lagunar del istmo de Tehuantepec, Oaxaca, México. Revista Mexicana de Biodiversidad 80:491-505.

MacArthur, R. H. 1965. Patterns of species diversity. Biological Review 40:510-533.

Macip-Ríos, R. y A. Muñoz-Alonso. 2008. Diversidad de lagartijas en cafetales y bosque primario en el Soconusco chiapaneco. Revista Mexicana de Biodiversidad 79:185-195.

Maclaurin, J. y K. Sterelny. 2008. What is biodiversity? The University of Chicago Press, Chicago. 224 p.

Molinari, J. 1989. A calibrated index for the measurement of evenness. Oikos 56:319-326.

Moreno, C. E. 2001. Métodos para medir la biodiversidad. M\&TManuales y Tesis SEA, vol. 1. Zaragoza, 84 p.

Moreno, C. E. y P. Rodríguez. 2010. A consistent terminology for quantifying species diversity? Oecologia 163:279-282.

Moreno, C. E. y P. Rodríguez. 2011. Commentary: Do we have a consistent terminology for species diversity? Back to basics and toward a unifying framework. Oecologia 167:889-892.

Pielou, E. C. 1980. Review on Grassle et al. (1979). Biometrics 36:742-743.

Podani, J. 2006. With a machete through the jungle: some thoughts on community diversity. Acta Biotheoretica 54:125-131.

Poole, R. W. 1974. An introduction to quantitative ecology. McGraw-Hill, New York. 532 p.

Ricotta, C. 2005. Trough the jungle of biological diversity. Acta Biotheoretica 53:29-38.

Samaniego-Gaxiola, J. A. y Y. Chew-Madinaveitia. 2007. Diversidad de géneros de hongos del suelo en 3 campos con diferente condición agrícola en La Laguna, México. Revista Mexicana de Biodiversidad 78:383-390.

Tokeshi, M. 1999. Species coexistence: ecological and evolutionary perspectives. Blackwell Sciences, Oxford. 454 p.

Tuomisto, H. 2010. A diversity of beta diversities: straightening up a concept gone awry. Part 1 . Defining beta diversity as a function of alpha and gamma diversity. Ecography 33:2-22.

Ulanowicz, R. E. 2001. Information theory in ecology. Computers and Chemistry 25:393-399.

Veech, J. A. y T. O. Crist. 2009. Partition: software for hierarchical partitioning of species diversity, version 3.0. http://www.users. muohio.edu/cristto/partition.htm; última consulta: 20.V.2010.

Veech, J. A. y T. O. Crist. 2010. Toward a unified view of diversity partitioning. Ecology 91:1988-1992.

Zar, J. H. 1999. Biostatistical analysis, cuarta edición. Prentice Hall, Upper Saddle River, New Jersey. 662 p. 